\title{
A rare complication of a rare disease: Amyloidosis in situs inversus
}

\author{
Abdullah Ozkok*, Mirac Vural Keskinler, Erhan Eken, Osman Kostek, Ozge Caklili, Alihan Oral, \\ Kubra Aydin Bahat, Omer Celal Elcioglu, Ali Bakan, Ali Riza Odabas \\ Istanbul Medeniyet University, Goztepe Training and Research Hospital, Nephrology Clinic, Istanbul, Turkey \\ Email: ${ }^{\text {abdullahozkok@yahoo.com }}$
}

Received 26 March 2013; revised 26 April 2013; accepted 4 May 2013

Copyright (C) 2013 Abdullah Ozkok et al. This is an open access article distributed under the Creative Commons Attribution License, which permits unrestricted use, distribution, and reproduction in any medium, provided the original work is properly cited.

\begin{abstract}
A 31-year-old female admitted with productive cough and progressively increasing edema in her legs. Four years ago she had the diagnosis of situs inversus (SI) totalis. She had $6.5 \mathrm{~g} /$ day proteinuria in 24 -hour urine analysis. High resolution computed tomography revealed bronchiectatic areas in the left lung. In renal biopsy, kongo and amyloid AA antibody positivity was detected in arteriolar walls and mesangial areas. The patient is currently being followed with ramipril and colchicine treatments. Primary ciliary dyskinesia (PCD) and resultant SI totalis are very rare anomalies characterized by the total inversion of all organs and chronic bronchiectasis. There are very few reports on the association of SI totalis with nephrotic syndrome. Patients with SI totalis and chronic bronchiectasis have increased risk of AA amyloidosis and possibly end stage renal disease and thus these patients should be regularly followed with proteinuria and serum creatinine levels.
\end{abstract}

Keywords: Situs Inversus; Amyloidosis; Bronchiectasis; Nephrotic Syndrome

\section{INTRODUCTION}

Primary ciliary dyskinesia (PCD) and resultant situs inversus (SI) totalis are very rare anomalies characterized by the total inversion of all abdominal and thoracic organs and chronic bronchiectasis [1]. The association of SI totalis with nephrotic syndrome has been reported only four times so far in which focal segmental glomerulosclerosis and amylodiosis were the etiologic factors [2-5]. Herein we report a case of nephrotic syndrome and amyloidosis due bronchiectasis in a patient with SI to talis.

\footnotetext{
"Corresponding author.
}

\section{CASE PRESENTATION}

A 31-year-old female patient admitted with fatigue, cough, sputum and edema in her legs progressively increasing in the last month. Four years ago she had the diagnosis of SI totalis and she had been followed for asthma with bronchodilatator therapy thereafter. In physical examination, she had bilateral soft pretibial edema, rhoncus and coarse crackles in the left lower and middle lung zones. Baseline biochemical laboratory data were presented in the Table 1. In urine analysis, +++ proteinuria and pyuria (7 leukocytes) were present and hematuria was absent. She had $6.5 \mathrm{~g} /$ day proteinuria in 24-hour urine analysis. HBsAg and Anti-HCV were negative. Serum complement levels $(C 3, C 4)$ were normal. High resolution computed tomography (HRCT) of the chest revealed cystic and cylindrical bronchiectatic areas with fibrotic sequel lesions (Figure 1). With the pre-diagnosis of amyloidosis due to bronchiectasis, rectal biopsy was performed. Amyloidosis was not detected in rectal biopsy. In renal biopsy, dense, homogeneous material deposition and kongo positivity were detected in arteriolar walls and mesangial areas. In histochemical analysis, strong positivity was detected with amyloid AA antibody. In echocardiography, cardiac involvement was not detected. The patient is currently being followed by nephrology polyclinics with the treatment of ramipril $10 \mathrm{mg}$ /day and colchicine $0.5 \mathrm{mg} 3$ times/day.

\section{DISCUSSION}

PCD is a genetically heterogeneous, autosomal recessive disorder characterized by ciliary dysfunction and impaired mucociliary clearance, resulting in a spectrum of clinical manifestations, including situs inversus (in approximately $50 \%$ of cases), chronic bronchitis leading to bronchiectasis, recurrent pneumonia, chronic rhinosinusitis, chronic otitis media, conductive hearing loss, hy- 
Table 1. Baseline biochemical laboratory data.

\begin{tabular}{lcc}
\hline & Values & Reference Values \\
\hline Creatinine & $0.7 \mathrm{mg} / \mathrm{dL}$ & $(0.7-1.4)$ \\
Calcium & $8.3 \mathrm{mg} / \mathrm{dL}$ & $(8.5-10.5)$ \\
Phosphorus & $4.5 \mathrm{mg} / \mathrm{dL}$ & $(2.7-4.5)$ \\
ALP & $62 \mathrm{U} / \mathrm{L}$ & $(30-135)$ \\
AST & $27 \mathrm{U} / \mathrm{L}$ & $(5-42)$ \\
ALT & $23 \mathrm{U} / \mathrm{L}$ & $(5-45)$ \\
LDH & $272 \mathrm{U} / \mathrm{L}$ & $(0-247)$ \\
GGT & $25 \mathrm{U} / \mathrm{L}$ & $(5-85)$ \\
Total bilirubin & $0.3 \mathrm{mg} / \mathrm{dL}$ & $(0.2-1.0)$ \\
Total cholesterol & $425 \mathrm{mg} / \mathrm{dL}$ & $(130-200)$ \\
LDL & $304 \mathrm{mg} / \mathrm{dL}$ & $(100-130)$ \\
Triglyceride & $259 \mathrm{mg} / \mathrm{dL}$ & $(60-150)$ \\
Total protein & $5 \mathrm{~g} / \mathrm{dL}$ & $(6.0-8.0)$ \\
Albumin & $2.1 \mathrm{~g} / \mathrm{dL}$ & $(3.2-5.5)$ \\
ESR & $84 \mathrm{~mm} / \mathrm{h}$ & $(0-20)$ \\
CRP & $0.39 \mathrm{mg} / \mathrm{dL}$ & $(0-0.8)$ \\
WBC & $13,400 / \mathrm{uL}$ & $(4000-11,000)$ \\
Hb & $13.1 \mathrm{~g} / \mathrm{dL}$ & $(12-18)$ \\
MCV & $88 \mathrm{fL}$ & $(80-100)$ \\
Platelet & $484,000 / \mathrm{uL}$ & $(150,000-400,000)$ \\
\hline
\end{tabular}

WBC: White blood cells, Hb: Hemoglobin, MCV: Mean corpuscular volume, ALP: Alkaline phosphatase, AST: Aspartat aminotransferase, ALT: Alanine aminotransferase, LDH: Lactate dehydrogenase, GGT: Gamma glutamyl transferase, LDL: Low density lipoprotein, ESR: Erythrocyte sedimentation rate, CRP: $\mathrm{C}$ reactive protein

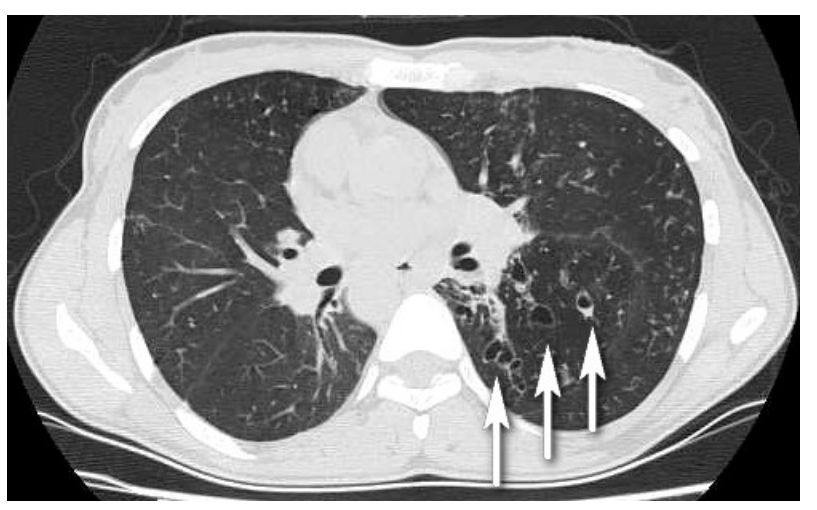

Figure 1. Dextrocardia and cystic, cylindrical bronchiectatic areas with fibrotic sequel lesions in the left lower and midzones (arrows).

drocephalus, retinitis pigmentosa and male infertility [1]. The incidence of PCD is estimated at 1/16,000 births based on prevalence of situs inversus and bronchiectasis [6].

Chronic productive cough is frequently reported in the majority of subjects $(84 \%-100 \%)$ because cough compensates for the lack of effective mucociliary clearance [7]. Impaired mucociliary clearance of the lower respiratory tract also leads to recurrent episodes of pneumonia or bronchitis. Clinical and radiographic evidence of bronchiectasis develops as the disease progresses. Review of HRCT findings of the lungs showed that bronchiectasis primarily involves the middle and lower lobes $(100 \%$ adults and $55 \%$ pediatric patients) [8].

The association of SI totalis with nephrotic syndrome has been reported only four times so far. Osman et al. [2] reported a case of amyloidosis due to bronchiectasis in a patient with SI similar to our case. This patient progressed to end stage renal failure (ESRD) and followed up under chronic hemodialysis treatment in contrast to our patient having normal renal functions. In the case by Vikrant et al. [4], the etiology of the nephrotic syndrome in such a patient was found to be focal segmental glomerulosclerosis. In a different report [5], authors discussed a case of SI with steroid sensitive nephrotic syndrome and in conclusion they suggested that disturbed trans TGF-beta and Smad signaling could be responsible for both nephrotic syndrome and situs inversus totalis. However, nephrotic syndrome in SI seems to be mostly related to amyloidosis secondary to bronchiectasis.

In conclusion, patients with SI totalis and chronic bronchiectasis have increased risk of AA amyloidosis and possibly ESRD. These patients should be regularly followed by proteinuria and serum creatinine tests and treated aggressively with angiotensin converting enzyme inhibitors and/or angiotensin receptor blocker treatments together with full-dose colchicine therapy to halt the progression to ESRD.

\section{REFERENCES}

[1] Leigh, M.W., Pittman, J.E., Carson, J.L., et al. (2009) Clinical and genetic aspects of primary ciliary dyskinesia/Kartagener syndrome. Genetics in Medicine, 11, 473487. doi:10.1097/GIM.0b013e3181a53562

[2] Osman, E.M., Abboud, O.I., Sulaiman, S.M., Musa, A.R., Beleil, O.M. and Sharfi, A.A. (1991) End-stage renal failure in Kartagener's syndrome. Nephrology Dialysis Transplantation, 6, 747. doi:10.1093/ndt/6.10.747

[3] Demir, M., Kutlucan, A. and Sezer, M.T. (2007) Renal amyloidosis in a patient with Kartagener syndrome. Renal Failure, 29, 117. doi:10.1080/08860220601039569

[4] Vikrant, S., Kumar, S., Raina, R. and Sharma, A. (2008) Nephrotic syndrome in a patient with situs inversus totalis. Clinical and Experimental Nephrology, 12, 215218. doi:10.1007/s10157-007-0027-0

[5] Domański, M., Domański, L. and Ciechanowski, K. (2005) Nephrotic syndrome and situs inversus viscerum: Correlation or coincidence. Journal of Nephrology, 18, 623-625.

[6] Katsuhara, K., Kawamoto, S., Wakabayashi, T. and Belsky, J.L. (1972) Situs inversus totalis and Kartagener's syndrome in a Japanese population. Chest, 61, 56-61. doi:10.1378/chest.61.1.56

[7] Noone, P.G., Leigh, M.W., Sannuti, A., et al. (2004) Primary ciliary dyskinesia: Diagnostic and phenotypic features. American Journal of Respiratory and Critical Care 
Medicine, 169, 459-467.

doi:10.1164/rccm.200303-365OC

[8] Kennedy, M.P., Noone, P.G., Leigh, M.W., et al. (2007) High-resolution $\mathrm{CT}$ of patients with primary ciliary dy- skinesia. American Journal of Roentgenology, 188, 1232 1238. doi:10.2214/AJR.06.0965

\section{LIST OF ABBREVATIONS}

SI: Situs inversus

PCD: Primary ciliary dyskinesia

HRCT: High resolution computed tomography

ESRD: End stage renal failure

TGF-beta: Transforming growth factor-beta

AA: Amyloid associated 